\title{
Barriers to effective diagnosis and management of a bleeding patient with undiagnosed bleeding disorder across multiple specialties: results of a quantitative case-based survey
}

\author{
This article was published in the following Dove Press journal: \\ Journal of Multidisciplinary Healthcare \\ 25 October 2012 \\ Number of times this article has been viewed
}

\author{
Mark T Reding' \\ David L Cooper ${ }^{2}$ \\ 'Center for Bleeding and Clotting \\ Disorders, University of Minnesota \\ Medical Center, Minneapolis, MN, \\ ${ }^{2}$ Medical Affairs, Novo Nordisk Inc, \\ Princeton, NJ, USA
}

\begin{abstract}
Background: Bleeding symptoms commonly seen by multiple physician specialties may belie undiagnosed congenital or acquired bleeding disorders. Acquired hemophilia is a potentially life-threatening cause of unexplained acute bleeding manifested by an abnormal activated partial thromboplastin time (aPTT) that does not correct with 1:1 mixing with normal plasma.

Methods: Practicing physicians (hematology/oncology, emergency medicine, geriatrics, internal medicine, rheumatology, obstetrics and gynecology, critical care medicine, and general surgery) completed an online survey based on a hypothetical case scenario.

Results: Excluding surgeons and obstetrician/gynecologist respondents, 302 physicians (about 50 per specialty) were presented with an older adult woman complaining of recurrent epistaxis. Nearly $90 \%$ ordered a complete blood count and coagulation studies (aPTT, prothrombin time $[\mathrm{PT}] /$ international normalized ratio [INR]). Despite a prolonged aPTT of 42 seconds, $<50 \%$ of nonhematologists would repeat the aPTT, and $<45 \%$ would consult a hematologist; emergency medicine physicians were least likely $(10 \%)$ and rheumatologists were most likely $(43 \%)$ to consult. After presentation weeks later with bruising and abdominal/back pain, $\geq 90 \%$ of physicians within each specialty ordered a complete blood count or PT/INR/aPTT. Despite an aPTT of 63 seconds, the majority did not repeat the aPTT. At this point, approximately $75 \%$ of internal medicine and geriatric physicians indicated they would consult a hematologist, versus $47 \%$ in emergency medicine and 50\% in critical care. All participants preferred abdominal computed tomography ( $80 \%-84 \%)$. After 12 hours of additional observation, $73 \%$ to $94 \%$ of respondents consulted a hematologist. Complete blood count revealed anemia and an aPTT twice the upper limit of normal; emergency medicine physicians remained least likely to request a consult.
\end{abstract}

Conclusion: Determining the cause of an abnormal coagulation study result should carry equal weight as looking for the site of bleeding and could be facilitated by consultation with a hematologist. Insight from this survey highlights knowledge and practice gaps that could be the target of focused educational initiatives.

Keywords: acquired hemophilia, hemorrhage, aPTT, partial thromboplastin time, coagulation disorders

\section{Introduction}

It is not uncommon to see bleeding symptoms in patients in outpatient or hospital-based practice. Older adult patients frequently have cutaneous bruising commonly diagnosed as senile purpura. Postpartum hemorrhage complicates pregnancy and accounts for significant morbidity and mortality, particularly in underdeveloped countries. ${ }^{1,2}$
Correspondence: Mark T Reding Code 480, 420 Delaware Street SE, Minneapolis, MN 55455, USA

$\mathrm{Tel}+\mathrm{l} 612625$ II04

Fax +l 6126256919

Email redin002@umn.edu 
Menorrhagia is a common clinical challenge and is often associated with secondary anemia, excessive fatigue, and a negative effect on health-related quality of life. ${ }^{3}$ Postoperative bleeding is one of the more common complications of surgery. Trauma is a leading cause of morbidity and mortality in the younger population. ${ }^{4}$

While bleeding symptoms may be commonly seen by physicians of multiple specialties, it is unclear how frequently these symptoms belie an underlying undiagnosed congenital or acquired bleeding disorder. In the US, the most common congenital bleeding disorders include von Willebrand disease, which affects approximately $1 \%$ of the population (males and females equally), ${ }^{5}$ and hemophilia A and B combined, which affect approximately 20,000 persons (essentially all males, with rare exception). ${ }^{6}$ Medications can also affect coagulation or platelet function, ${ }^{7}$ as can certain herbal supplements. ${ }^{8}$ Trauma and surgery can lead to blood loss, and critical reduction in coagulation factors can lead to additional nonsurgical bleeding complications (coagulopathic bleeding). ${ }^{9}$

The most ubiquitous method for evaluating coagulation is prothrombin time (PT)/international normalized ratio (INR) and activated partial thromboplastin time (aPTT). Typically, they are ordered to monitor anticoagulant therapy (PT/INR for warfarin, aPTT for heparin), to evaluate coagulation preoperatively, or in response to hemorrhagic symptoms. The PT/INR provides an assessment of the extrinsic (tissue factor-dependent) and final common pathways of the coagulation cascade, while the aPTT provides an assessment of the intrinsic (tissue factor-independent) and final common pathways. $^{10}$

An example of a potentially life-threatening cause of unexplained recent onset or acute bleeding associated with a prolonged aPTT is acquired hemophilia, with an incidence of approximately 1 to 4 per million/year. ${ }^{11}$ Acquired hemophilia primarily affects older adults, ${ }^{12}$ an ever-growing segment of the population that presents for medical evaluation and care, but may also occur during pregnancy as postpartum hemorrhage or in association with other underlying diseases, including cancer and autoimmune disorders. ${ }^{13}$ Prompt diagnosis is a primary determinant of prognosis in acquired hemophilia ${ }^{14}$ because initiation of definitive therapy (ie, hemostatic and immunosuppressive) is delayed until the diagnosis is made. Acquired hemophilia-related bleeding does not respond to the typical management algorithms used for hemorrhaging in a patient and, therefore, is associated with high morbidity and mortality. Severe bleeds occur in up to $90 \%$ of patients with acquired hemophilia, ${ }^{11}$ and the reported overall mortality rate in these patients ranges from $8 \%$ to $22 \% .{ }^{15-17}$ Given the rarity of acquired hemophilia, combined with the general lack of familiarity of nonhematologists with this condition, the diagnosis of acquired hemophilia poses a clinical challenge, even in patients presenting with straightforward bleeding and an isolated, prolonged aPTT.

A survey was conducted of physicians across a number of specialties to identify potential barriers to the effective recognition and management of this rare but important cause of serious bleeding. The survey, based on an actual case found to be the result of acquired hemophilia, focused on participants' stepwise evaluation and management of a case patient who presented to the hospital with recent-onset bleeding. The survey also assessed participants' history with regard to consulting hematologists, discovering or diagnosing underlying bleeding disorders, and encountering acquired hemophilia in clinical practice. Findings pertaining to the interpretation and follow-up of abnormal coagulation studies, enlistment of hematology consultation, and the diagnostic decision process in an actively bleeding case patient were the primary areas assessed in this analysis. Results were evaluated across specialties to determine any specialty-specific practice trends that might hinder effective recognition and management of an actively bleeding patient with coagulopathy, including acquired hemophilia.

\section{Materials and methods}

Physicians within the specialties of hematology, hematology/ oncology, emergency medicine, geriatrics, internal medicine, rheumatology, obstetrics and gynecology, critical care medicine, and general surgery were randomly sampled from the American Medical Association Physician Masterfile. Physicians who were part of the Harris Interactive Online Physician Panel were invited via email to participate in the survey, while physicians who were not part of the panel were invited via first class mail. The invitation provided a uniform resource locator address and password for one-time use to log on to the survey site, where invitees first encountered questions to determine eligibility to participate in the survey.

Eligible physicians were required to be actively practicing, with additional specialty-specific requirements. In particular, hematologists, hematologist/oncologists, general surgeons, and emergency medicine and critical care physicians were required to be practicing at an acute care hospital. For internists and geriatricians, at least $60 \%$ of their practice had to consist of patients older than 60 years of age. Obstetricians/gynecologists were required to have an active obstetrics practice. Upon confirmation of eligibility, participants completed the online survey, the average duration of which was approximately 
10 minutes. All surveys were completed over a 2-week period (January 14-28, 2010). The study was designed to assess diagnostic barriers within each specialty. As such, each specialty was recruited to a final sample of 50 complete responses to ensure a sufficient number of responses to generate reasonable hypotheses about specialists' behavior to test in face-to-face interviews.

Findings from the surveys completed by the physicians specializing in hematology, hematology/oncology, emergency medicine, geriatrics, internal medicine, rheumatology, and critical care medicine are presented herein. General surgeons and obstetrics and gynecology physicians each completed different surveys from the aforementioned specialties; the findings of those surveys are not included here.

Survey questions focused on the diagnostic and general therapeutic approach to the management of a case patient (Figure 1) whose presentation and clinical course were based on those of a real patient who experienced significant delays in diagnosis despite the involvement of multiple specialists. At each juncture in the case, participants were given lists of actions that included diagnostic tests (laboratory, radiology, other), consultations, and potential treatments addressing bleeding or anemia (local hemostasis, transfusion). Since familiarity with the upper limits of normal of the PT/ aPTT tests was being tested in this study, upper limits of normal were not specified, and aPTTs were $\geq 25 \%$ to $30 \%$ above the upper limits of normal and set at a value that respective specialties would recognize as abnormal based on their experience with ordering and interpreting this laboratory test. The survey did not specify whether laboratory testing, imaging studies, or consultations in this hypothetical case had to be available immediately or locally, and the assumption was that respondents would answer based on their best medical judgment in an ideal practice situation.

The case summary and questions were tailored in a limited way for certain specialties. Rheumatologists were only asked about an initial emergency department/urgent care presentation because it was expected that if a bleeding disorder developed in one of their patients it would present in an outpatient or emergency department/urgent care setting. Conversely, critical care specialists started at presentation 2, since their involvement in a recurrent epistaxis workup would be unlikely. Vital signs were adjusted to make it more likely that a potential intensive care unit admission would require their evaluation. Since emergency medicine physicians would most likely have ordered an imaging study initially to define the patient's retroperitoneal hematoma, we allowed for the option of a general surgery consultation even though the computed tomography findings were never presented.
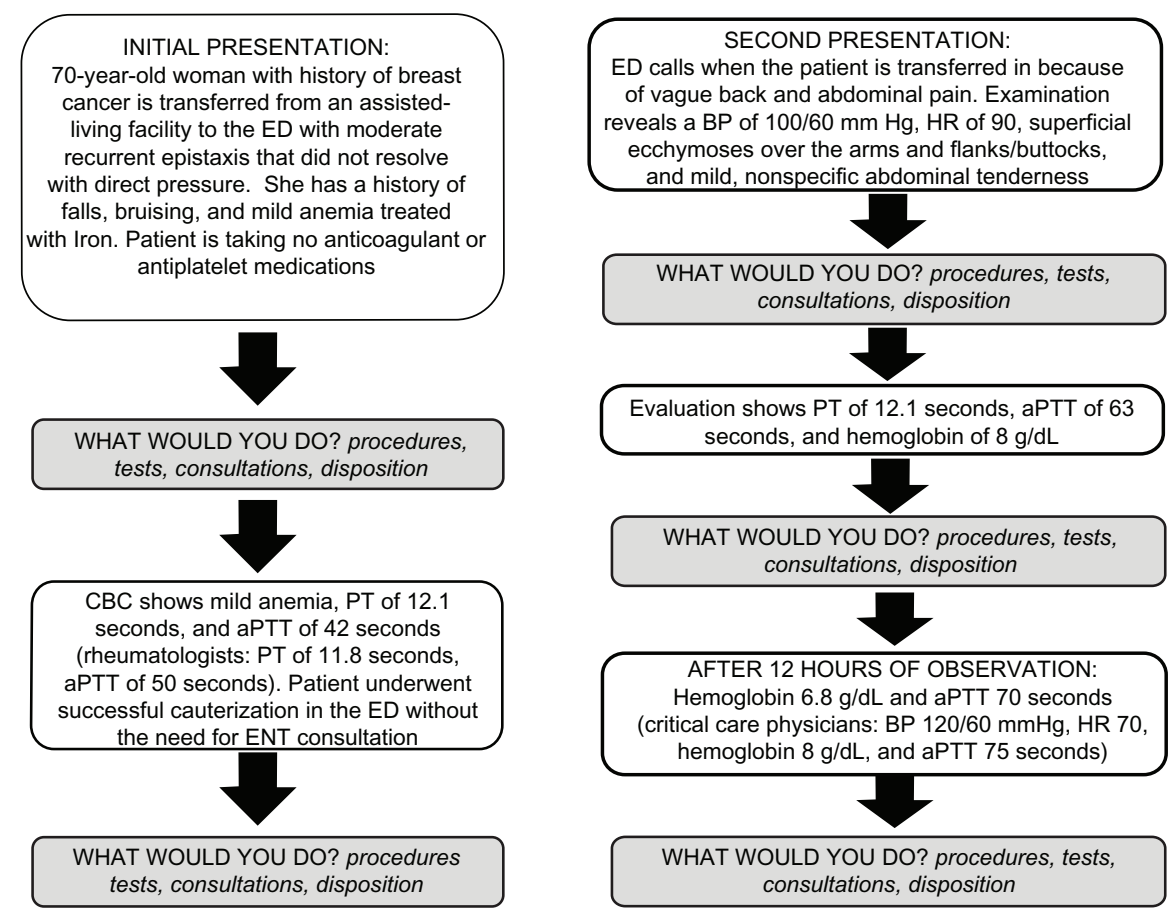

Figure I Schematic of survey content. The case directed to participating critical care specialists began with the second presentation. In contrast, rheumatologists were presented with only the initial patient presentation and subsequent laboratory findings. Where indicated, unique laboratory values and/or clinical parameters were incorporated into the cases presented to participating rheumatologists and critical care specialists.

Abbreviations: aPTT, activated partial thromboplastin time; BP, blood pressure; CBC, complete blood count; ED, emergency department; ENT, ear, nose, throat specialty; $H R$, heart rate; PT, prothrombin time. 
Hematology/oncology respondents were given the option of ordering additional specialty coagulation laboratory tests (eg, mixing studies).

Participants were additionally questioned about their experiences diagnosing underlying bleeding disorders and, in the case of nonhematologist specialists, consulting hematologist colleagues. Conversely, hematology and hematology/ oncology specialists were asked about being on the receiving end of consultations, specifically from emergency medicine personnel, and the reasons for those consultations.

\section{Results}

A total of 302 physicians (50-51 per specialty) participated in the case-based survey reported herein. Demographics of the specialty groups were consistent: mean age ranged from 45.6 to 50.6 years, and mean years of practice experience ranged from 14.5 to 19.3. Detailed demographic information about the participants is shown in Table 1.

\section{Initial presentation: recommended diagnostic evaluations and hematology consultation}

Faced with an older adult female patient with recurrent epistaxis, nearly $90 \%$ of physicians in each of the surveyed specialties indicated they would have ordered a complete blood count and coagulation studies (PT/INR and aPTT) as part of the initial evaluation (Figure 2A). Despite abnormal results of the aPTT at 42 seconds (with an upper limit of normal typically ranging from 35 to 39 seconds, although not specified in the case), less than half the physicians in most specialties would have chosen to repeat the coagulation studies as one of the next steps (Figure 2B). In contrast, $67 \%$ of hematologists would have repeated these studies. Less than $45 \%$ of surveyed physicians in all nonhematology specialties would have consulted a hematologist after reviewing the initial coagulation study results (Figure $2 \mathrm{~B}$ ). Emergency medicine physicians were least likely to obtain a consult (10\%). Rheumatologists were most likely to obtain a consult (43\%), although they were presented with an initial aPTT that was more abnormal (50 seconds) than the aPTT initially presented to the other specialists.

\section{Second presentation: recommended diagnostic evaluations and hematology consultation}

After the patient's second presentation several weeks later with bruising and abdominal/back pain, again nearly $90 \%$ or more of respondents (including critical care specialists,

Table I Demographic characteristics of survey participants

\begin{tabular}{|c|c|c|c|c|c|c|}
\hline \multirow[t]{2}{*}{ Characteristic } & \multicolumn{6}{|l|}{ Specialty } \\
\hline & $\begin{array}{l}\text { Hematology } \pm \\
\text { oncology } \\
(n=5 I)\end{array}$ & $\begin{array}{l}\text { Emergency } \\
\text { medicine } \\
(n=50)\end{array}$ & $\begin{array}{l}\text { Geriatrics } \\
(n=50)\end{array}$ & $\begin{array}{l}\text { Internal } \\
\text { medicine } \\
(n=50)\end{array}$ & $\begin{array}{l}\text { Rheumatology } \\
(n=5 I)\end{array}$ & $\begin{array}{l}\text { Critical } \\
\text { care medicine } \\
(n=50)\end{array}$ \\
\hline Mean age (years) & 48.9 & 47.2 & 45.6 & 49.1 & 50.6 & 46.6 \\
\hline Mean duration in practice (years) & 18.7 & 16.1 & 14.5 & 19.1 & 19.3 & 16.2 \\
\hline \multicolumn{7}{|l|}{ Practice description (\%) } \\
\hline Mostly office or clinic based & 53 & 6 & 28 & 81 & 96 & 2 \\
\hline Mostly/exclusively hospital or laboratory based & 14 & 93 & 8 & 6 & 4 & 75 \\
\hline Equally hospital and office/clinic based & 33 & 0 & 26 & 13 & 0 & 21 \\
\hline Mostly long-term-care facility based & 0 & 0 & 32 & 0 & 0 & 0 \\
\hline Mostly hospice based & 0 & 1 & 2 & 0 & 0 & 0 \\
\hline Other & 0 & 0 & 3 & 0 & 0 & 2 \\
\hline \multicolumn{7}{|l|}{ Office/clinic description ${ }^{a}(\%)$} \\
\hline Solo practice & 3 & 100 & 2 & 22 & 21 & 30 \\
\hline Single-specialty practice & 68 & 0 & 65 & 23 & 44 & 35 \\
\hline Multispecialty practice & 28 & 0 & 34 & 54 & 34 & 44 \\
\hline \multicolumn{7}{|l|}{ Hospital affiliation (\%) } \\
\hline Academic/tertiary care medical center & 39 & 14 & 32 & 17 & 18 & 36 \\
\hline Private/tertiary care hospital & 27 & 22 & 18 & 19 & 30 & 22 \\
\hline Community/primary care hospital & 34 & 60 & 40 & 63 & 51 & 37 \\
\hline VA/government facility & 0 & 3 & 9 & I & 0 & 5 \\
\hline Mean no. patients seen in a typical week & 77.1 & 105.9 & 75.7 & 114.8 & 97.1 & 67.9 \\
\hline
\end{tabular}

Notes: "Base respondents consist of only those who practiced in an office or clinic setting at the time of the survey. Therefore, for the emergency and critical care medicine specialties, this base would have been extremely small $(n=2$ and $n=10$, respectively).

Abbreviation: VA, Veterans Administration. 
A

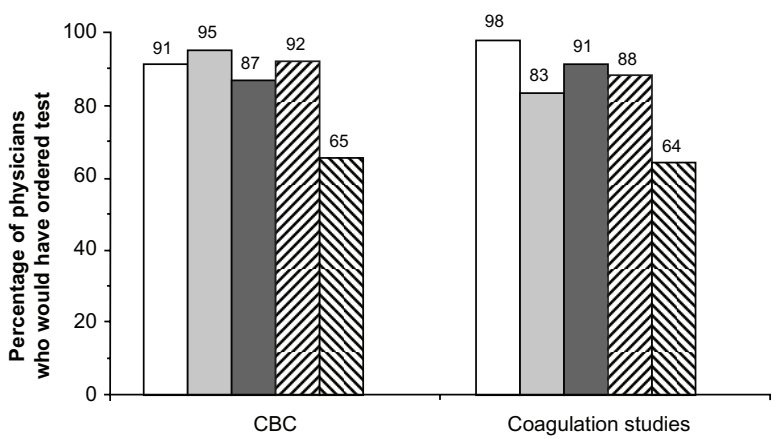

C

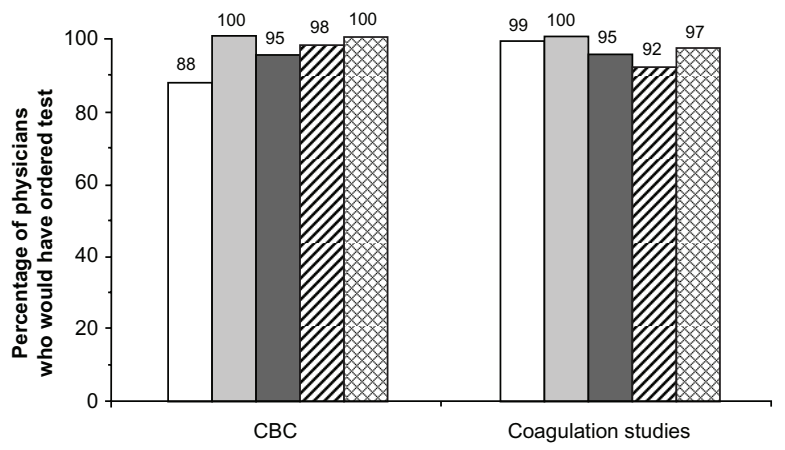

B

After initial laboratory tests

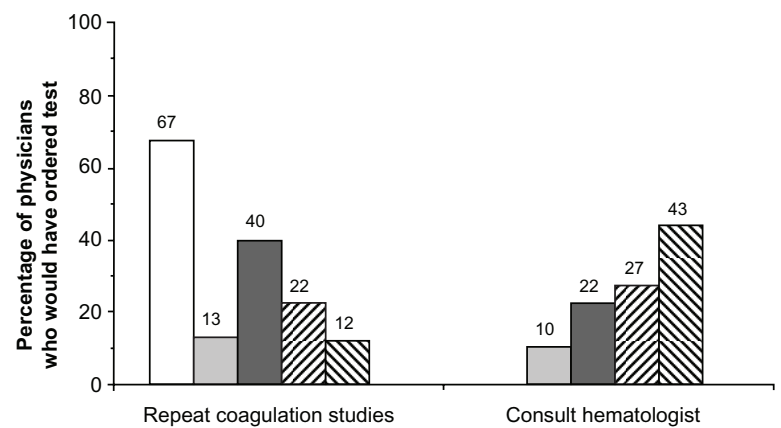

D After second laboratory tests

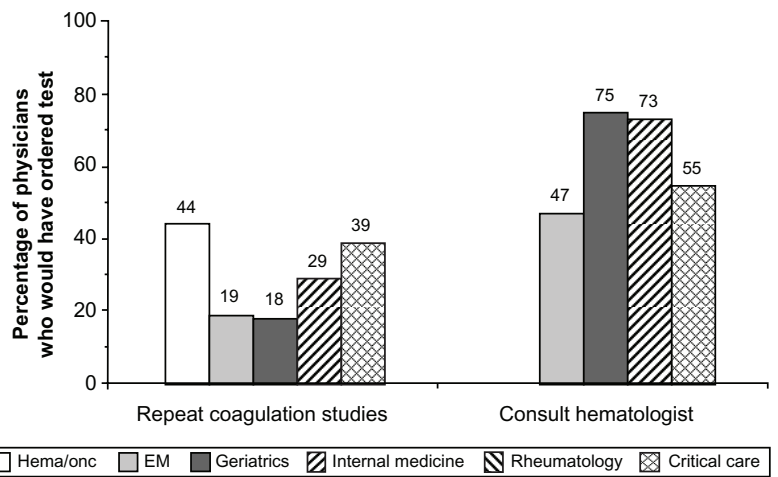

Figure 2 Recommendations for laboratory tests and hematology consultation across surveyed specialties: first and second presentations. The percentages of physicians in each specialty who would have ordered specific hematologic laboratory tests after each of the case patient's presentations (A and C) and the percentages who would have ordered repeat coagulation studies and consulted a hematologist after reviewing each set of laboratory results (B and $\mathbf{D})$ are shown. Critical care specialists began their case presentation with the second presentation and were given a different set of vital signs from the other specialties (see Figure I). Rheumatologists were presented with only the initial patient presentation and subsequent laboratory findings, which included PT and aPTT values that were different from those provided to the other specialties (see Figure I). In addition, the case patient presented to rheumatologists was evaluated and managed on an outpatient basis. Hematologists were not queried about consulting a hematologist in response to hematologic laboratory results.

Abbreviations: CBC, complete blood count; EM, emergency medicine; heme/onc, hematology/oncology.

who began their case review at this juncture in the patient's clinical course) would have ordered both a complete blood and coagulation studies as part of their initial evaluation (Figure 2C). When these results revealed a clearly abnormal and markedly changed aPTT of 63 seconds, the majority of respondents indicated they still would not have repeated coagulation studies (Figure 2D). Approximately $75 \%$ of internal medicine and geriatrics physicians would have consulted a hematologist at this point, compared with $47 \%$ and $50 \%$ of emergency medicine and critical care specialists, respectively (Figure 2D). Sixty percent of hematologists and hematologists/oncologists surveyed would have evaluated the patient's peripheral blood smear at this point.

In addition to the aforementioned hematologic laboratory tests, other diagnostic tests and consultations that participants would have recommended from a series of options as part of their evaluation after the patient's second presentation are shown in Figure 3. Participants across specialties clearly preferred computed tomography of the abdomen (80\%-84\%) over abdominal ultrasound (9\%-28\%), upper gastrointestinal endoscopy $(2 \%-16 \%)$, or colonoscopy $(0 \%-16 \%)$. Emergency medicine physicians demonstrated the greatest breadth of testing, with $82 \%$ additionally recommending urinalysis and $92 \%$ recommending a stool guaiac test. Requests for gastroenterology consultations ranged from $10 \%$ to $43 \%$ and were highest for the internal medicine group, which also had the highest proportion of physicians recommending endoscopy (16\% each for upper gastrointestinal endoscopy and colonoscopy).

For the purposes of assessing multiple specialties, the next 12 hours of observation in the case patient's clinical course were described as having occurred in the emergency department. By the end of this observation period, the aPTT had further increased to at least twice the upper limit of normal, while the hemoglobin level had decreased. Internists and geriatricians were most inclined to repeat the laboratory studies at this juncture (Figure 4). In contrast with previous time points, by 


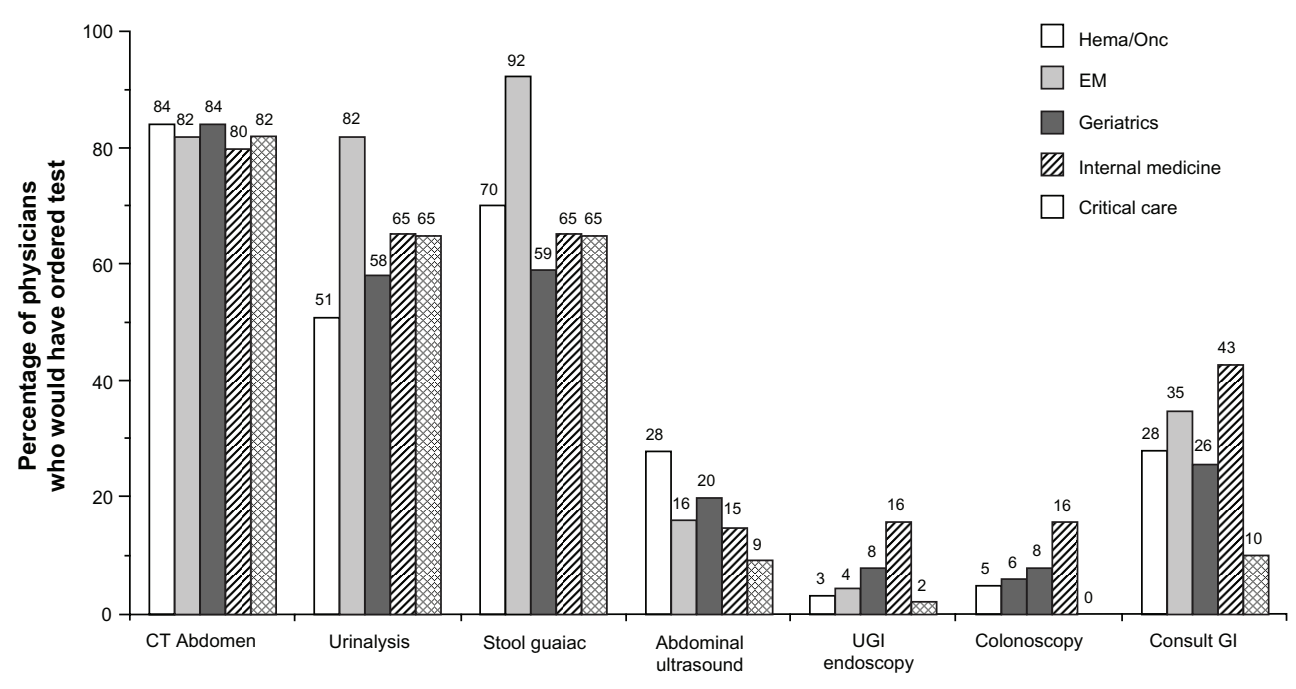

Figure 3 Additional tests and consultations recommended after second clinical presentation.

Notes: Percentages of physicians who would have included the respective tests or consultations as part of their evaluation after the case patient's second clinical presentation are shown. Additional tests that physicians could have selected included platelet function tests, renal ultrasound, upper and lower gastrointestinal series, and "other," all of which were chosen by $50 \%$ or fewer of physicians across specialties.

Abbreviations: CT, computerized tomography; EM, emergency medicine; GI, gastroenterology; UGl, upper gastrointestinal.

this point in the patient's clinical presentation, $73 \%$ to $94 \%$ of respondents would have consulted a hematologist. Emergency medicine physicians remained least likely to obtain a consult. The majority of hematologists would have ordered 1:1 mixing studies of patient and normal plasma to rule out coagulation

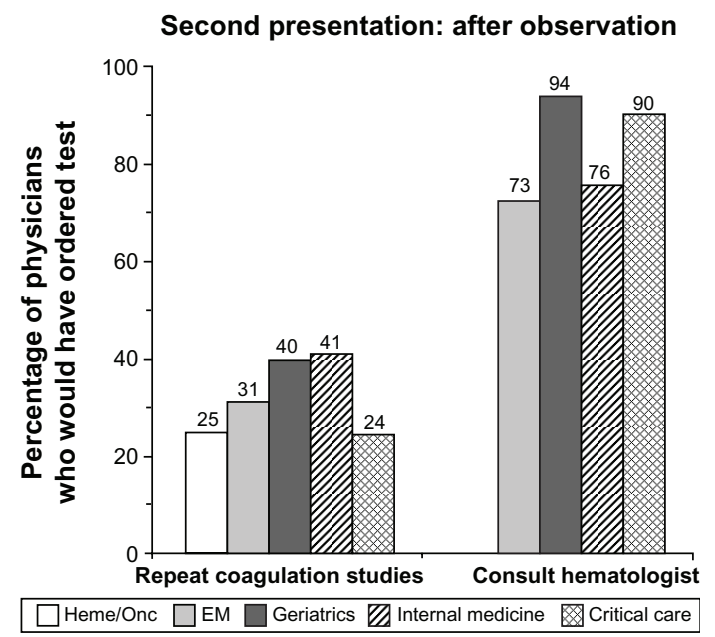

Figure 4 Recommendations for repeat coagulation studies and hematology consultation in response to laboratory results after 12 hours of observation subsequent to the case patient's second presentation.

Notes: The percentages of physicians within each specialty who would have repeated the coagulation studies and consulted hematology in response to the patient's laboratory results after 12 hours of observation are shown. Laboratory results at this point were remarkable for a further decrease in hemoglobin (to $6.8 \mathrm{~g} / \mathrm{dL}$ [or $8 \mathrm{~g} / \mathrm{dL}$ for the critical care physicians]) and increase in aPTT (to 70 seconds [or 75 seconds for the critical care physicians]).

Abbreviations: EM, emergency medicine; heme/onc, hematology/oncology. inhibitors (97\%) and fibrinogen/fibrin split products testing (75\%) in response to the laboratory results obtained after 12 hours of observation, as part of a diagnostic evaluation of the prolonged aPTT. Emergency medicine physicians were given the additional option of consulting general surgery practitioners; $2 \%$ would have done so after the first set of laboratory results at the second presentation, and $20 \%$ would have done so in response to the second set of laboratory results.

\section{Recommendations regarding case patient disposition}

The percentages of physicians in each specialty who would have recommended admission of this case patient to a general hospital floor or back to a skilled nursing facility or nursing home after initial presentation with subsequent abnormal aPTT of 42 seconds are shown in Figure 5A. Across the majority of specialties, less than $20 \%$ of physicians would have recommended overnight admission to a general hospital floor, while more than $50 \%$ of physicians in these specialties would have recommended discharge to a skilled nursing facility or a nursing home. In contrast with respondents from other specialties, a slightly higher proportion of hematologists and hematologist/oncologists would have endorsed a higher level of care at this point; $36 \%$ of physicians in this group indicated they would have favored overnight hospital admission over discharge to a skilled nursing facility. 
A

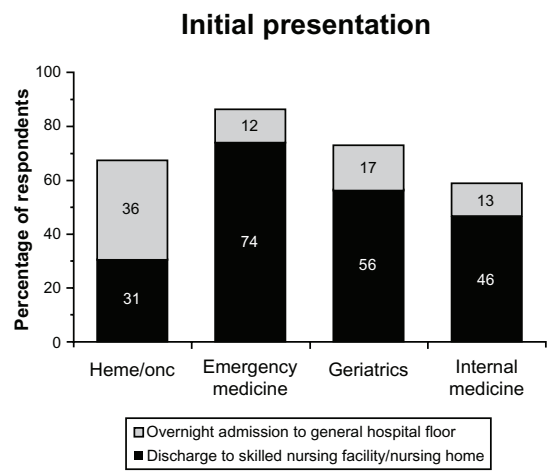

B

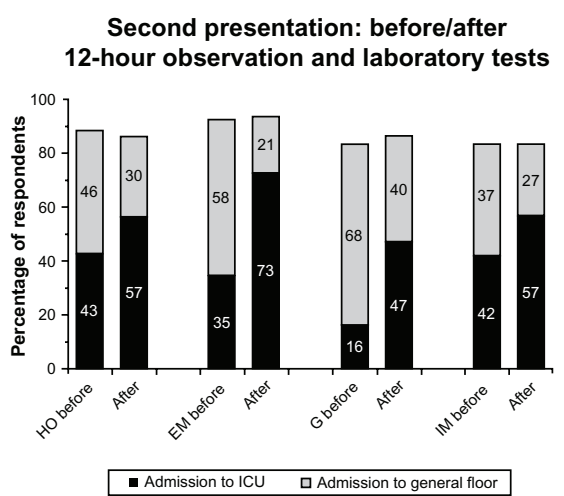

Figure 5 Recommended disposition after initial and second presentations and after 12 hours of observation. (A) The proportion of physicians in each surveyed specialty who would have recommended overnight admission to the general hospital floor or discharge to a skilled nursing care facility/nursing home after the case patient's first presentation and subsequent laboratory results are shown. (B) The percentage of physicians in each specialty recommending admission to the general hospital floor versus the ICU after the patient's initial laboratory results upon second admission and subsequent results obtained after 12 hours of observation are shown. See Figure I for the summarized laboratory findings.

Abbreviations: EM, emergency medicine; G, geriatrics; HO, hematology/oncology; ICU, intensive care unit; IM, internal medicine.

The majority of physicians faced with the second presentation would have recommended that the patient be admitted to the hospital. The differences among specialties were most noticeable in allocation to a general hospital floor versus an intensive care unit after review of initial laboratory results or the second set of laboratory results obtained after 12 hours of observation (Figure $5 \mathrm{~B}$ ). While approximately $80 \%$ or more of physicians in each specialty would have recommended hospital admission in response to both sets of laboratory results, the proportion recommending admission specifically to the intensive care unit increased as the laboratory values deteriorated (worsening anemia and coagulopathy). Based on the laboratory results obtained after 12 hours of observation, nearly $50 \%$ or more of physicians in each specialty would have

\section{A}

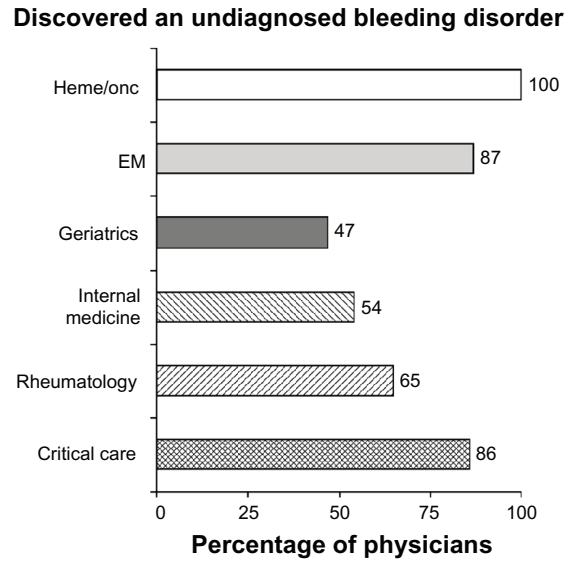

recommended admission to the intensive care unit in lieu of a general hospital floor, compared with approximately $40 \%$ or less across specialties after the initial laboratory results obtained during the patient's second presentation. The difference was most noticeable for physicians in emergency medicine (35\%-73\%), the specialty most likely to refer the patient to an inpatient medical/hospitalist or critical care service.

\section{Familiarity with bleeding disorders}

More than $85 \%$ of emergency medicine and critical care physicians reported having ever discovered an underlying bleeding disorder, while $100 \%$ of hematologists and hematologist/oncologists reported having ever diagnosed one (Figure 6A). The percentage of physicians in other specialties

\section{B}

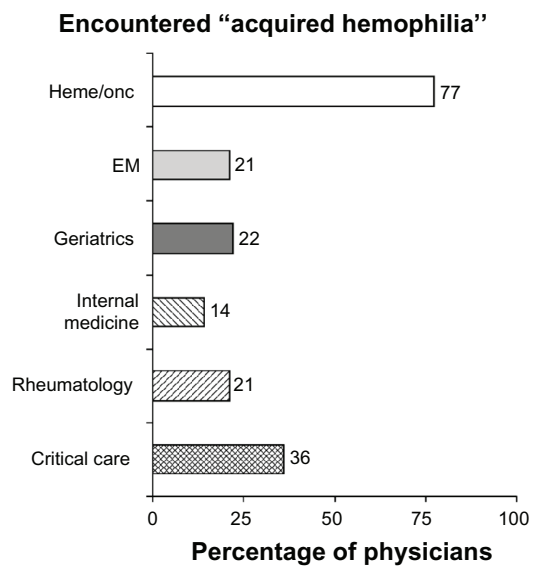

Figure 6 Prior physician experience with bleeding disorders. Results represent (A) the percentage of physicians in each specialty that had ever discovered (or diagnosed, in the case of the hematologists and hematologist/oncologists) an underlying bleeding disorder and (B) the percentages of those physicians who had specifically encountered "acquired hemophilia."

Abbreviations: EM, emergency medicine; heme/onc, hematology/oncology. 
who had ever discovered an underlying bleeding diathesis ranged from $47 \%$ to $65 \%$. Among those physicians who had previously diagnosed or discovered a bleeding disorder, 14\% to $77 \%$ had specifically encountered "acquired hemophilia" (Figure 6B), although this quantitative survey did not assess whether they understood this disorder. Hematologists and hematologists/oncologists accounted for the highest percentage in this group, while internal medicine specialists accounted for the lowest percentage. Nearly $40 \%$ of critical care specialists reported having previously encountered acquired hemophilia.

\section{History of hematology consultations}

Table 2 shows the frequencies with which various specialties had ever consulted a hematologist when they encountered a patient with an abnormal PT/INR and aPTT and no history of a bleeding diathesis or medications that affect coagulation. The distribution of reasons for which respondents would have consulted a hematologist is also shown in Table 2. In the majority of specialties (emergency medicine, geriatrics, and internal medicine), the highest percentage of physicians had consulted a hematologist 1 to 2 times for a patient with an unexplained prolonged PT/INR or aPTT. In contrast, more than $80 \%$ of rheumatologists had consulted a hematologist 3,4 , or 5 or more times for the same reason. The highest percentage (39\%) of critical care physicians had consulted a hematologist at least
5 times for such a patient. The most common reason for consulting a hematologist was abnormal coagulation study results in the setting of clinical bleeding (Table 2), which was parallel to the findings for the 100 additional surgeons and obstetricians surveyed (data not shown). The most common response among critical care specialists to the open-ended question, "What are the circumstances around which you would call for a hematology consult?" was "an unexplained abnormal lab or bleeding" (33 like or similar mentions), followed by "any unexplained bleeding disorder" (6 like or similar mentions).

Conversely, the frequency with which hematologists had ever been consulted by an emergency medicine provider for an abnormal PT/INR or aPTT in a patient with no history of bleeding diathesis or medications that affect coagulation is outlined in Table 2 . The majority (57\%) of hematologists had been consulted a minimum of 5 times by their colleagues in emergency medicine. The majority of these consults pertained to significantly abnormal coagulation studies with or without clinical bleeding (Table 2).

\section{Discussion}

Bleeding is commonly encountered in outpatient and hospital-based medical practice and can have a wide variety of underlying causes. While bleeding may be relatively common, most acquired and congenital bleeding disorders are uncommon, and some, such as acquired hemophilia,

Table 2 Utilization of and rationale for hematology consultation

\begin{tabular}{|c|c|c|c|c|c|c|}
\hline & \multicolumn{6}{|l|}{ Specialty } \\
\hline & $\begin{array}{l}\text { Emergency } \\
\text { medicine }\end{array}$ & Geriatrics & $\begin{array}{l}\text { Internal } \\
\text { medicine }\end{array}$ & Rheumatology & $\begin{array}{l}\text { Critical care } \\
\text { medicine }\end{array}$ & $\begin{array}{l}\text { Hematology } \pm \\
\text { oncology }\end{array}$ \\
\hline \multicolumn{7}{|l|}{ Frequency of hematology consultations ${ }^{\mathrm{a}}(\%)$} \\
\hline Never & 16 & 24 & 25 & 7 & 5 & - \\
\hline I-2 times & 46 & 35 & 41 & 11 & 30 & - \\
\hline $3-4$ times & 28 & 23 & 23 & 42 & 26 & - \\
\hline $5+$ times & 11 & 19 & 12 & 40 & 39 & - \\
\hline \multicolumn{7}{|l|}{$\begin{array}{l}\text { Frequency of consultations received from } \\
\text { the emergency department }{ }^{\mathrm{a}}(\%)\end{array}$} \\
\hline Never & - & - & - & - & - & I \\
\hline $\mathrm{I}-2$ times & - & - & - & - & - & 32 \\
\hline $3-4$ times & - & - & - & - & - & 10 \\
\hline $5+$ times & - & - & - & - & - & 57 \\
\hline \multicolumn{7}{|l|}{ Reasons for hematology consultation ${ }^{\mathrm{b}}(\%)$} \\
\hline $\begin{array}{l}\text { Any coagulation study outside of normal } \\
\text { ranges but not significantly abnormal }\end{array}$ & 0 & 0 & 0 & 12 & - & 61 \\
\hline Any significantly abnormal coagulation studies & 34 & 55 & 54 & 42 & - & 85 \\
\hline $\begin{array}{l}\text { Combination of abnormal coagulation } \\
\text { studies with clinical bleeding }\end{array}$ & 87 & 78 & 78 & 65 & - & 88 \\
\hline
\end{tabular}

Notes: aSurvey participants were asked whether they had ever requested (or received) a hematology consultation for abnormal PT or aPTT in a patient with no history of bleeding diathesis or medications that affect coagulation.

bSummated percentages may exceed 100\% because of potential overlap among possible responses.

Abbreviations: aPTT, activated partial thromboplastin time; PT, prothrombin time. 
are rare. Nevertheless, the consequences of failure to recognize promptly and treat properly a bleeding disorder may be significant. ${ }^{18}$ In the case of acquired hemophilia, morbidity and mortality rates are particularly high: severe bleeding is experienced by up to $90 \%$ of affected patients, and mortality rates are as high as $22 \% .{ }^{11}$ This survey provided a step-wise methodology to tease out specialty-specific patterns of interpretation of clinical data to identify barriers to the diagnosis and treatment of underlying bleeding disorders. The sample size obtained across specialties was sufficient to generalize these findings, at least to the point of identifying specific issues for education and development of clinical decisionmaking pathways.

When presented with a clinical picture that includes a recent history or symptoms of active bleeding, clinicians typically obtain coagulation times, such as the PT/INR and aPTT, as part of the initial diagnostic evaluation. Proper interpretation of laboratory test results includes recognition of abnormal values and, more important, the potential clinical significance of such results. A common pitfall in the interpretation of coagulation times is failure to appreciate that even mildly abnormal values may represent a serious underlying coagulation deficit. Another important observation is to identify how an abnormal laboratory value may have changed over time, which can be facilitated by the ability of electronic medical record systems to display data trends. In the absence of iatrogenic causes, even a mildly elevated PT/ INR or aPTT may be indicative of true coagulopathy and should not be ignored or dismissed, particularly when there is evidence of bleeding, as was the case with this patient, even at initial presentation.

After excluding laboratory error, the differential diagnosis of an isolated prolongation of aPTT includes heparin effect, lupus anticoagulant, and deficiency of or antibody against an intrinsic pathway factor (VIII, IX, XI, or XII). ${ }^{19,20}$ A detailed history, focusing on factors such as heparin exposure, ${ }^{19,20}$ history of thromboembolism (lupus anticoagulant), ${ }^{21}$ and prior personal or family history of bleeding, ${ }^{22}$ may provide diagnostic clues. Laboratory testing should include a 1:1 mixing test of patient plasma with control plasma to determine whether a prolonged aPTT is the result of an intrinsic pathway factor deficiency or an inhibitor that continues to block the activity of the intrinsic system even in the presence of control plasma. The majority of inhibitor antibodies identified in this manner will turn out to be lupus anticoagulants. Although far less common, this is the same diagnostic pathway that leads to the identification of the antifactor VIII antibodies associated with acquired hemophilia. ${ }^{23}$ Unlike acquired hemophilia, lupus anticoagulants typically do not present with bleeding, and the abnormal aPTT is due to interference with phospholipid-dependent coagulation reactions. Once an acquired antifactor VIII antibody is suspected, confirmatory testing includes measuring factor VIII activity, which should be significantly reduced, and the Bethesda assay, ${ }^{24}$ which is used to quantitate antifactor VIII antibodies inhibitor activity.

We found a general lack of appropriate consideration and response to the presenting symptom of bleeding and the prolonged aPTT throughout this case study. This is consistent with data from the European Acquired Haemophilia Registry (EACH-2), which reported a median delay of 3 days between onset of bleeding symptoms and the diagnosis of acquired hemophilia and a median delay of one day between the first abnormal aPTT test in those same patients and the established diagnosis. ${ }^{23}$ In addition, we found that emergency medicine and critical care physicians were reluctant to consider a bleeding disorder as the primary explanation for this patient's clinical presentation. The disposition of a patient with active hemorrhage and evidence of coagulopathy should be based on several factors, including the patient's current condition and anticipated clinical course, taking into account the presenting vital signs and evolving laboratory findings. At the time of the patient's second presentation, vital signs were notable for mild tachycardia and a pulse pressure at the upper limit of normal, and subsequent laboratory findings indicated a decreasing hemoglobin level and an increasing aPTT. These findings alone prompted hospital admission, although the exact location (general floor versus intensive care unit) of admission may vary, based on the level of monitoring and nurse-to-patient ratios in a particular hospital. Another important variable is the anticipated potential for clinical deterioration, which is based in large part on clinician appreciation of the seriousness of the diagnosis. We found a consistent tendency to consider admission to a general floor bed with the second presentation, even though this was ultimately an unstable, critically ill patient with an undiagnosed bleeding disorder. This survey clearly highlights several pitfalls in the optimal management of acquired hemophilia.

We also found that the physicians who participated in this survey were reluctant to consult a hematologist as they worked through this case scenario, particularly given that options for additional testing (liver function, disseminated intravascular coagulation) were not available to evaluate for common causes of coagulopathy. This was particularly true of emergency medicine and critical care specialists. Relative to their reported historical experience with hematology consultations during an 
average practice experience of approximately 20 years, this survey finding was somewhat surprising. Rheumatologists and critical care specialists reported a greater frequency of hematology consultation relative to the other specialties (Table 2). We would expect the emergency medicine physicians to be most likely to consult a hematologist, yet $16 \%$ of them reported never having consulted a hematologist. The highest percentage (46\%) had only consulted a hematologist 1 or 2 times, and almost one quarter of geriatricians and internists had never consulted a hematologist, even though these specialists would be expected to first encounter patients with undiagnosed bleeding disorders, including acquired hemophilia. One potential reason for not seeking hematology consultation might be the lack of availability of hematology/ oncology specialists with expertise in coagulation disorders, including in rural and community hospitals.

A limitation of this survey was that one cannot interpret the thinking behind the responses of the individual participants. Therefore, 31 qualitative 45-minute interviews were conducted subsequent to the quantitative study and focused particularly on critical care $(n=7)$, emergency medicine $(n=6)$, hematology/ oncology $(n=4)$, or hematology $(n=2)$ physicians to understand the reasoning behind their decisions and depth of knowledge (unpublished data). We found that the physicians' focus was generally on finding the source (location) of bleeding and not on finding the underlying reason for bleeding. This could potentially lead to surgical intervention in the face of an underlying bleeding disorder, with subsequent adverse outcomes. In a series of 67 patients with acquired hemophilia at a single center in Bonn, Germany, 4 of 5 deaths were the result of surgical intervention for bleeding at outside hospitals in the setting of a delayed diagnosis of acquired hemophilia. ${ }^{13}$

When queried about their experience encountering and/ or diagnosing underlying bleeding disorders, particularly acquired hemophilia, more than $85 \%$ of physicians in hematology, hematology/oncology, emergency medicine, and critical care medicine reported having ever discovered or diagnosed an underlying bleeding disorder, compared with 65\%, 54\%, and $47 \%$ of rheumatologists, internists, and geriatricians, respectively. While reports of ever specifically having encountered acquired hemophilia were high, it is unclear from this study whether the participants truly understood the diagnosis. This seems unlikely, given the rarity of acquired hemophilia, relative to the reported frequency of having encountered it. Except for hematology and/or oncology (77\%) and critical care (36\%) specialists, approximately one quarter of surveyed physicians had ever encountered acquired hemophilia. Although they accounted for the highest percentage of physicians who had ever encountered this condition, nearly one quarter of hematologists had never encountered acquired hemophilia. Subsequent unpublished data from the aforementioned qualitative research further suggest that, compared with hematology practitioners, specialists in hematology/oncology, who likely practice mostly oncology, might be able to identify "mixing studies" and "inhibitors" but might not fully understand the underlying pathophysiology that constitutes acquired hemophilia, making it hard for them to recognize the condition. Given the survey findings reflecting the infrequency with which most physicians have encountered these conditions, consultation with a hematologist may facilitate the diagnostic evaluation and proper management of a hemorrhaging patient suspected of having an underlying bleeding diathesis, particularly acquired hemophilia.

The consulting hematologist can provide specific guidance, leading to the prompt diagnosis and optimal management of an actively bleeding patient with acquired hemophilia, including initiation of immunosuppression, which is usually necessary to eradicate the inhibitor and to prevent additional bleeding episodes. However, this requires a level of familiarity and expertise in treating acquired hemophilia and other rare bleeding disorders that is not often seen outside of an academic hematology practice. This represents yet another barrier to the effective diagnosis and management of this rare yet serious bleeding diathesis.

\section{Conclusion}

For the hospitalist or intensivist charged with the care of a bleeding patient, immediate stabilization is the initial priority and should take precedence over determination of a specific etiology of bleeding. However, in the presence of underlying coagulopathy, particularly acquired hemophilia or other rare disorders, traditional measures of stabilization may not be as effective as expected. A prolonged PT/INR or aPTT in the absence of iatrogenic causes should never be ignored, even with only minimally prolonged values, and determination of the cause of an abnormal coagulation study should carry at least equal weight to looking for the anatomic site of bleeding. Any delay in establishing the diagnosis of a bleeding diathesis such as acquired hemophilia can result in significant morbidity or even death. While hospitalists and intensivists should be able to conduct a thorough differential of bleeding and eliminate most common etiologies, consultation with a hematologist (particularly one with specific expertise in coagulation disorders) may facilitate the evaluation of coagulopathic patients and subsequent interpretation of diagnostic findings, as well as initiation of appropriate treatment. 
Given the rarity of acquired hemophilia, as exemplified by the findings of this survey, physicians must harbor a high index of suspicion to diagnose this condition promptly in patients who present with recent-onset or acute bleeding. Given the high morbidity and mortality, it is especially important to identify acquired hemophilia. The insights from this survey highlights knowledge and practice gaps that could be the focus of targeted educational initiatives, including diagnostic algorithms, to ensure proper and efficient workup of the abnormal laboratory studies that characterize acquired hemophilia.

\section{Disclosure}

MR is a consultant for and DC is an employee of Novo Nordisk Inc, the company that sponsored this study and manufactures a product approved for treatment of bleeding episodes in acquired hemophilia. The quantitative and qualitative research surveys described in this paper were completed by Harris Interactive, New York, NY, under funding provided by Novo Nordisk Inc, Princeton, NJ. A descriptive analysis of the results was prepared by Harris Interactive. The case study that forms the basis for the market research is based on an actual case treated by MR. Writing and editorial assistance was provided by Lara Primak, MD, of Ethos Health Communications in Newtown, PA, with financial support from Novo Nordisk Inc, in compliance with international guidelines for good publication practice.

\section{References}

1. Khan KS, Wojdyla D, Say L, Gulmezoglu AM, Van Look PF. WHO analysis of causes of maternal death: a systematic review. Lancet. 2006; 367:1066-1074.

2. Shakur H, Elbourne D, Gulmezoglu M, et al. The WOMAN Trial (World Maternal Antifibrinolytic Trial): tranexamic acid for the treatment of postpartum haemorrhage: an international randomised, double blind placebo controlled trial. Trials. 2010;11:40.

3. El-Nasher SA, Hopkins MR, Barnes SA, et al. Health-related quality of life and patient satisfaction after global endometrial ablation for menorrhagia in women with bleeding disorders: a follow-up survey and systemic review. Am J Obstet Gynecol. 2010;202:348.e1-e7.

4. Patton GC, Coffey C, Sawyer SM, et al. Global patterns of mortality in young people: a systematic analysis of population health data. Lancet. 2009;374:881-892.

5. Bowman M, Hopman WM, Rapson D, Lillicrap D, Silva M, James P. A prospective evaluation of the prevalence of symptomatic von Willebrand disease (VWD) in a pediatric primary care population. Pediatr Blood Cancer. 2010;55:171-173.
6. Siddiqi A-e-A, Ebrahim SH, Soucie JM, Parker CS, Atrash HK. Burden of disease resulting from hemophilia in the US. Am J Prev Med. 2010; 38:S482-S488.

7. Rice TW, Wheeler AP. Coagulopathy in critically ill patients: part 1 : platelet disorders. Chest. 2009;136:1622-1630.

8. Ang-Lee MK, Moss J, Yuan C-S. Herbal medicines and perioperative care. J Am Med Assoc. 2001;286:208-216.

9. McGilvray ID, Rotstein OD. Assessment of coagulation in surgical critical care patients. In: Holzheimer RG, Mannick JA, editors. Surgical Treatment: Evidence-Based and Problem-Oriented. Munich, Germany: Zuckschwerdt; 2001.

10. Kamal AH, Tefferi A, Pruthi RK. How to interpret and pursue an abnormal prothrombin time, activated partial thromboplastin time, and bleeding time in adults. Mayo Clin Proc. 2007;82:864-873.

11. Franchini M, Lippi G. Acquired factor VIII inhibitors. Blood. 2008;112: $250-255$.

12. Baudo F, de Cataldo F. Acquired hemophilia in the elderly. In: Balducci L, Ershler W, de Gaetano G, editors. Blood Disorders in the Elderly. Cambridge, UK: Cambridge University Press; 2008.

13. Franchini M, Gandini G, Di Paolantonio T, Mariani G. Acquired hemophilia A: a concise review. Am J Hematol. 2005;80:55-63.

14. Zeitler H, Ulrich-Merzenich G, Goldmann G, Vidovic N, Brackmann H-H, Oldenburg J. The relevance of the bleeding severity in the treatment of acquired hemophilia - an update of a single-centre experience with 67 patients. Haemophilia. 2010;16:95-101.

15. Green D, Lechner K. A survey of 215 non-hemophilic patients with inhibitors to factor VIII. Thromb Haemost. 1981;45:200-203.

16. Hay CRM, Negrier C, Ludlam CA. The treatment of bleeding in acquired haemophilia with recombinant factor VIIa: a multicentre study. Thromb Haemost. 1997;78:1463-1467.

17. Morrison AE, Ludlam CA, Kessler C. Use of porcine factor VIII in the treatment of patients with acquired hemophilia. Blood. 1993;81: 1513-1520.

18. Collins PW, Hirsch S, Baglin TP, et al. Acquired hemophilia A in the United Kingdom: a 2-year national surveillance study by the United Kingdom Haemophilia Centre Doctors' Organisation. Blood. 2007;109: 1870-1877.

19. Seegmiller A, Sarode R. Acquired bleeding disorders. In: Irwin RS, Rippe JM, editors. Irwin and Rippe's Intensive Care Medicine. 6th ed. Philadelphia, PA: Lippincott Williams \& Wilkins; 2008.

20. Wheeler AP, Rice TW. Coagulopathy in critically ill patients: part 2: soluble clotting factors and hemostatic testing. Chest. 2010;137: 185-194.

21. Shapiro SS. The lupus anticoagulant/antiphospholipid syndrome. Annu Rev Med. 1996;47:533-553.

22. Huth-Kuhne A, Baudo F, Collins P, et al. International recommendations on the diagnosis and treatment of patients with acquired hemophilia A. Haematologica. 2009;94:566-575.

23. Collins PW, Percy CL. Advances in the understanding of acquired haemophilia A: implications for clinical practice. Br J Haematol. 2010; 148:183-194.

24. Verbruggen B, Novakova I, Wessels H, Boezeman J, van den Berg M, Mauser-Bunschoten E. The Nijmegen modification of the Bethesda assay for factor VIII:C inhibitors: improved specificity and reliability. Thromb Haemost. 1995;73:247-251.
Journal of Multidisciplinary Healthcare

\section{Publish your work in this journal}

The Journal of Multidisciplinary Healthcare is an international, peerreviewed open-access journal that aims to represent and publish research in healthcare areas delivered by practitioners of different disciplines. This includes studies and reviews conducted by multidisciplinary teams as well as research which evaluates the results or conduct of such teams or

\section{Dovepress}

healthcare processes in general. The journal covers a wide range of areas and welcomes submission from practitioners at all levels, from all over the world. The manuscript management system is completely online and includes a very quick and fair peer-review system. Visit http://www.dovepress.com/testimonials.php to read real quotes from published authors. 